\title{
A 3.1 10.6 GHz CMOS Cascaded Two-stage Distributed Amplifier for Ultra-Wideband Application
}

\author{
Kuan-Hung Chen and Chorng-Kuang Wang \\ Graduate Institute of Electronics Engineering, and Department of Electrical Engineering, \\ National Taiwan University, 106 Taipei, Taiwan R. O. C. \\ E-mail: ckwang@cc.ee.ntu.edu.tw
}

\begin{abstract}
In this paper, a CMOS cascaded two-stage distributed amplifier for ultra-wideband (UWB) application is presented. The circuit using two-stage cascaded topology achieves better gain-bandwidth product performance than conventional CMOS distributed amplifiers. The simulated gain is $18 \mathrm{~dB}$ with $\pm 1 \mathrm{~dB}$ gain flatness over $3.1 \sim 10.6 \mathrm{GHz}$ bands. Input and output are matched to $50 \Omega$, and the retum losses of input and output are below $-10 \mathrm{~dB}$ and $-9 \mathrm{~dB}$ respectively. The power dissipation is $54 \mathrm{~mW}$ with $1.8 \mathrm{~V}$ power supply. The circuit was fabricated in $0.18-\mu \mathrm{m} 1 \mathrm{P} 6 \mathrm{M}$ RF CMOS process.
\end{abstract}

\section{INTRODUCTION}

UWB is an emerging technology for wireless communication. Unlike narrow-band system, UWB has capability for high data rate communication applications, such as imaging systems, vehicular radar systems, and ground penetrating [1]. Although the standard of UWB has not been finalized, many proposed researches target at the band between 3.1 and $10.6 \mathrm{GHz}$. In this work, the design goal is to achieve an amplifier with gain of $18 \mathrm{~dB}$ over 3.1 to $10.6 \mathrm{GHz}$ [12].

Distributed amplification, characterized by wide bandwidth, has been prevalent over sixty years. Lots of distributed amplifiers are fabricated on the compound semiconductor processes for applications of mini-meter wave. However, the CMOS technology is less expensive than compound semiconductor processes and has higher potential for integration with the digital part, especially for silicon-on-chip solutions. Researches on the CMOS distributed amplifiers have made considerable progress in the recent years.

Of these researches, one accomplished a gain of $5 \pm 1.2 \mathrm{~dB}$ from $300 \mathrm{kHz}$ to $3 \mathrm{GHz}$, and $4.7 \mathrm{GHz}$ unity-gain cutoff frequency [2]. In another work, a 0.5 to $8.5 \mathrm{GHz}$ CMOS fully differential distributed amplifier was realized with a gain of $5.5 \pm 1.5 \mathrm{~dB}$ in a $0.6-\mu \mathrm{m}$ CMOS process [4]. Lastly, a 0.5 to 14 $\mathrm{GHz}$ with $10.6 \pm 0.9 \mathrm{~dB}$ gain and a 0.6 to $22 \mathrm{GHz}$ with $7.3 \pm$ $0.8 \mathrm{~dB}$ gain distributed amplifier using $0.18-\mu \mathrm{m}$ CMOS RF process were proposed [7]-[8]. Among these, amplifier's gain does not exceed $10.6 \mathrm{~dB}$, which is not high enough for advance applications, such as UWB system. The cascaded single-stage distributed amplifier (CSDA) topology was brought out for increasing the amplifier's gain [6]. In that work, the CSDA has $21 \mathrm{~dB}$ gain and $5 \mathrm{GHz}$ bandwidth but the CSDA needs more complicated $\mathrm{dc}$-wise setup than conventional distributed amplifiers.

In this paper, a cascaded two-stage distributed amplifier (CTDA) is proposed, and it possesses the high gain property of CSDA without complicate dc-wise setup. This paper is organized as follows. Some basics of the conventional distributed amplifier design are described in Section II. Section III covers the topology of CTDA and some comparisons between the conventional distributed amplifier, CSDA and CTDA. Section IV shows the simulation results, and conclusions are given in section $\mathrm{V}$.

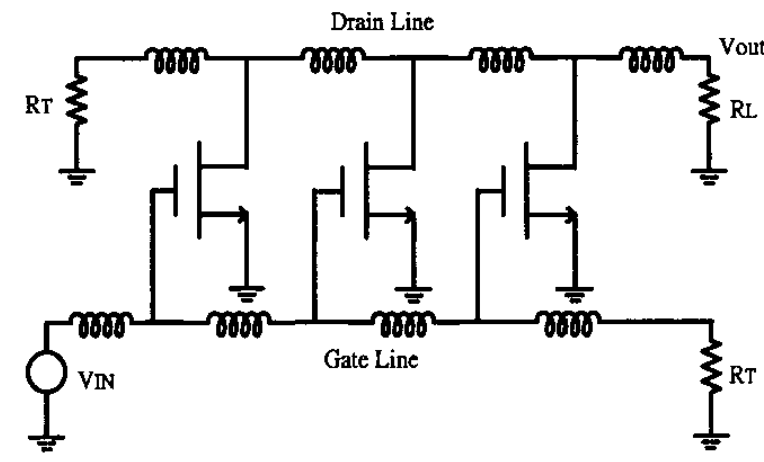

Fig. 1 Conventional distributed amplifier

\section{BASICS OF DISTRIBUTED AMPLIFIER DESIGN}

Distributed amplifiers employ the topology that several gain stages are in parallel and series-inductors are introduced to separate capacitances of the input and output node of adjacent gain stages. This topology builds two artificial transmission lines, gate line and drain line. Output current is combined additively by the current flowing from each gain stage. The additive characteristic results in a relatively low gain, while the distributed nature of capacitance can achieve very wide bandwidth. Fig. 1 shows a three-stage conventional distributed amplifier. Design equations can be referred to [10], [11]. The voltage gain can be written as [11]: 


$$
A_{\nu}=-\frac{g_{m}}{2 \sqrt{1-\left(\omega / \omega_{c}\right)^{2}}} \sqrt{\frac{L}{C}} e^{-\frac{N}{2}\left(\theta_{d}+\theta_{g}\right)} \frac{\sinh N \frac{\left(\theta_{d}-\theta_{g}\right)}{2}}{\sinh \frac{\left(\theta_{d}-\theta_{g}\right)}{2}}
$$

where

$\begin{array}{ll}N & \text { number of stages; } \\ g_{m} & \text { gain stage transconductance; } \\ \omega_{c} & \text { cutoff frequency of transmission lines; } \\ \theta_{g} & \text { propagation constant of gate line; } \\ \theta_{d} & \text { propagation constant of drain line; }\end{array}$

The cutoff frequency $\left(\omega_{c}\right)$ of transmission line is $2 / \sqrt{L C}$. It can be derived that voltage gain becomes maximum when $\theta_{g}=$ $\theta_{d}$, as shown in Fig. 2. The more the propagation constant of the gate line differs from that of drain line, the more the amplifier gain decays.

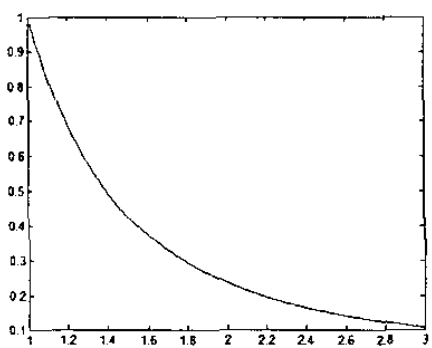

Fig. 2 Normalized gain vs. Ratio of $\theta_{g}$ and $\theta_{d}$

When $\theta_{g}=\theta_{d,}$ the equation of voltage gain can be simplified

$$
A_{V}=-\frac{N \cdot g_{m}}{2 \sqrt{1-\left(\omega / \omega_{c}\right)^{2}}} \sqrt{\frac{L}{C}} e^{-N \theta}
$$

Intuitively, the voltage gain can be enhanced when large $N$ is selected. Because the transmission lines are not ideal, some parasitic effects such as spiral inductor parasitic resistance make the propagation constant not purely imaginary, which leads to path loss. The voltage gain is not proportional to the number of stages anymore but achieves a maximum when the optimal value of $N$ is used. The equation of optimal $N$ is written as [11]:

$$
N_{o p t}=\frac{\ln \frac{\theta_{r d}}{\theta_{r g}}}{\theta_{r d}-\theta_{r g}}
$$

where

$\theta_{r g} \quad$ real part of propagation constant of gate line;

$\theta_{r d} \quad$ real part of propagation constant of drain line;

For CMOS process, the $N_{\text {opt }}$ is between 3 to 5 . This is one of the substantial reasons that distributed amplifier gain can not be large.
In [6], the CSDA is proposed to enhance its gain. Assuming all the load resistance of each gain stage are the same, the equation of gain is:

$$
A_{V}=\left(\frac{1}{2} g_{m} \cdot R_{o}\right)^{N}
$$

where

$N$ number of stages cascaded;

$\mathrm{g}_{\mathrm{m}} \quad$ gain stage transconductance;

$R_{O} \quad$ output resistance of each stage;

This is a novel approach to increase the gain of the amplifier. The gain grows in proportional to the power of $N$. However, if the gain of each stage $\left(g_{m} \cdot R_{o} / 2\right)$ is not large, the amplifier gain can not grow very fast. In addition, extra components such as RF chock may be needed to setup dc operating voltage at each inter-connected node.

Therefore, we propose the cascaded two-stage distributed amplifier (CTDA) to solve these problems. Next session discusses the CTDA circuit topology.

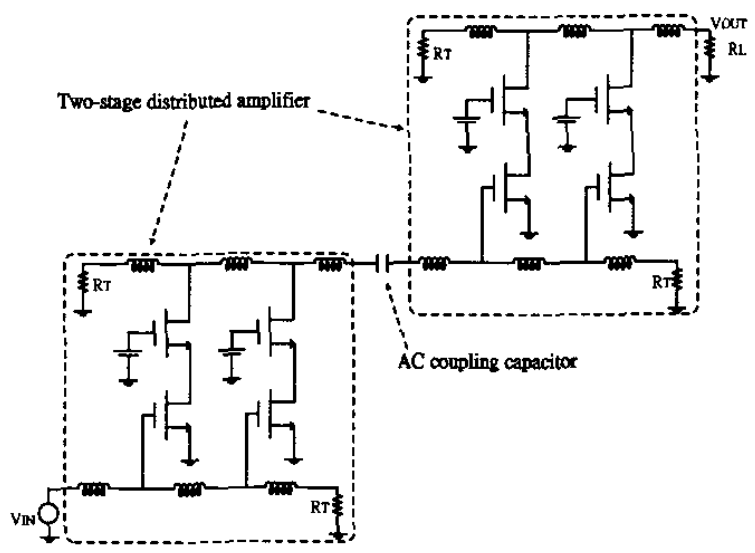

Fig. 3 Cascaded two-stage distributed amplifier

\section{CTDA TOPOLOGY AND GAIN ENHANCEMENT}

The CTDA circuit topology is shown in Fig. 3. It could be regarded as two conventional two-stage distributed amplifiers cascaded. AC coupling capacitor is used for connecting these two conventional distributed amplifiers and DC setup can be accomplished in the same way as that of conventional distributed amplifier. The cascode gain cell configuration is used for its high maximum available gain, better input-output isolation, and high output impedance [8]. The gain of CTDA can be written as:

$$
A_{y}=\left(\frac{1}{2} \cdot 2 \cdot g_{m} \cdot R_{o}\right)^{N}
$$

where

$N$ number of two-stage distributed amplifier cascaded; 
Assuming conventional distributed amplifier, CSDA and CTDA are all composed of four gain stages, the demanded transconductance per gain stage can be plotted respectively in different required gain, as shown in Fig. 4 . When required gain is between $12 \mathrm{~dB} \sim 24 \mathrm{~dB}$, the transconductance of CTDA is the smallest among three of them. Power dissipation of the circuit is usually in proportion to the needed transconductance per gain-stage, so CTDA consumes the least power.

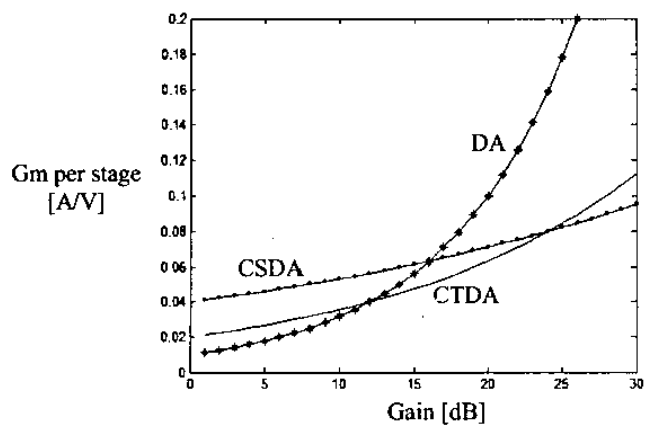

Fig. 4 Transconductance per stage versus Target gain

\section{Simulation RESULTS}

The CMOS CTDA was simulated by Advanced Design System (ADS). Fig. 5 and Fig. 6 show the S-parameters and the noise figure of the CTDA. The gain $\left(\mathrm{S}_{21}\right)$ is $18 \mathrm{~dB}$ with $1.2 \mathrm{~dB}$ ripple from $3.1 \mathrm{GHz}$ to $10.6 \mathrm{GHz}$. The input and output return losses are less than $-10 \mathrm{~dB}$ and $-9 \mathrm{~dB}$ respectively. The reverse isolation $\mathrm{S}_{12}$ is $40 \mathrm{~dB}$ or better over the whole bandwidth. The noise figure is between $5 \mathrm{~dB}$ and $7 \mathrm{~dB}$. Power consumption of the CTDA is only $54 \mathrm{~mW}$. Die photo is shown in Fig. 7. Table 1 summarizes the performance of the CTDA. The recently reported performances of CMOS distributed amplifiers compared with this work are summarized in Table 2. This work demonstrates the highest gain-bandwidth production (GBP).

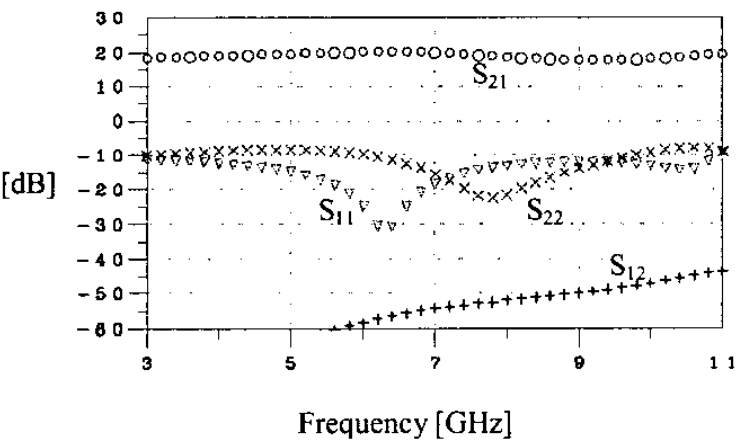

Fig. 5 Simulated S-parameters

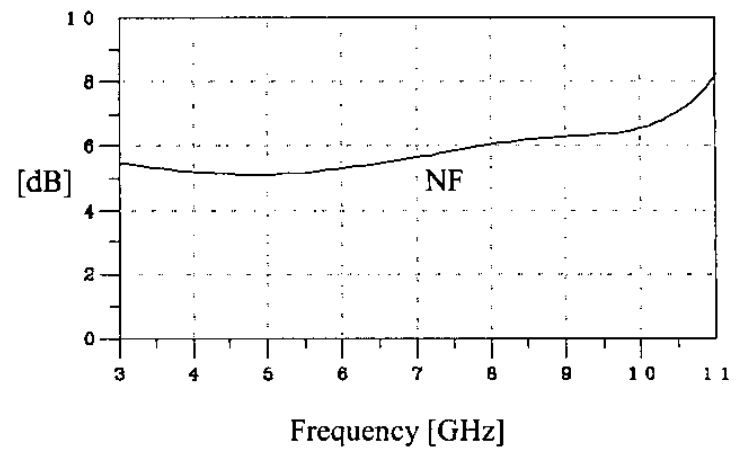

Fig. 6 Simulated Noise figure

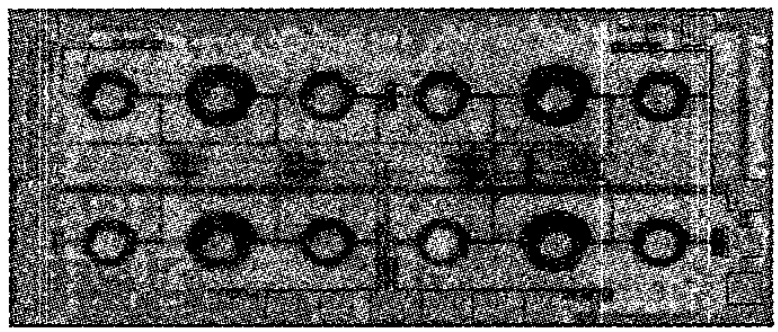

Fig. 7 Die photo of CTDA

\begin{tabular}{|c|c|}
\hline & Simulation Results \\
\hline Technology & TSMC 0.18 um \\
\hline Power Supply & $1.8 \mathrm{~V}$ \\
\hline Frequency & $3.1 \sim 10.6 \mathrm{GHz}$ \\
\hline Gain $\left(\mathrm{S}_{21}\right)$ & $18 \mathrm{~dB}(1.2 \mathrm{~dB}$ ripple $)$ \\
\hline Input Return loss $\left(\mathrm{S}_{11}\right)$ & $<-10 \mathrm{~dB}$ \\
\hline Output Retum loss $\left(\mathrm{S}_{22}\right)$ & $<-9 \mathrm{~dB}$ \\
\hline Isolation( $\left.\mathrm{S}_{12}\right)$ & $<-40 \mathrm{~dB}$ \\
\hline Noise Figure & $5 \sim 7 \mathrm{~dB}$ \\
\hline Power Consumption & $54 \mathrm{~mW}$ \\
\hline Area & $2.2 \mathrm{~mm} \mathrm{x} 1 \mathrm{~mm}$ \\
\hline
\end{tabular}

Table 1 Performance summary of CTDA

\section{CONCLUSION}

A CMOS cascaded two-stage distributed amplifier has been proposed. The conventional distributed amplifier, CSDA and CTDA have been compared under similar conditions. The CTDA can achieve a voltage gain of $12 \sim 24 \mathrm{~dB}$ using less power consumption than those two. In this work, an $18 \mathrm{~dB}$ voltage gain CTDA consuming only $54 \mathrm{~mW}$ has been presented for UWB application. Comparing with other previously published CMOS DAs, this work demonstrates the highest gain-bandwidth production. 


\begin{tabular}{|c|c|c|c|c|c|c|c|c|}
\hline Reference & Process & $\begin{array}{c}\text { Bandwidth } \\
(\mathrm{GHz})\end{array}$ & $\begin{array}{c}\text { Gain } \\
(\mathrm{dB})\end{array}$ & $\begin{array}{c}\text { GBP } \\
(\mathrm{GHz})\end{array}$ & $\begin{array}{c}\text { S11 } \\
(\mathrm{dB})\end{array}$ & $\begin{array}{c}\text { S22 } \\
(\mathrm{dB})\end{array}$ & $\begin{array}{c}\text { VDD } \\
(\mathrm{V})\end{array}$ & $\begin{array}{c}\text { Power Consumption } \\
(\mathrm{mW})\end{array}$ \\
\hline$[3]$ & $0.6 \mu \mathrm{m} \mathrm{CMOS}$ & 4 & 6.5 & 8.5 & -7 & -10 & 3 & 83.4 \\
\hline$[4]$ & $0.6 \mu \mathrm{m} \mathrm{CMOS}$ & 7.5 & 5.5 & 14 & -6 & -9.5 & 3 & 216 \\
\hline$[6]$ & $0.35 \mu \mathrm{m} \mathrm{CMOS}$ & 5 & 21 & 56 & -10 & -10 & 2.2 & 132 \\
\hline$[9]$ & $0.18 \mu \mathrm{m} \mathrm{CMOS}$ & 10 & 8 & 25 & - & - & - & - \\
\hline$[7]$ & $0.18 \mu \mathrm{m} \mathrm{CMOS}$ & 22 & 7.3 & 51 & -8 & -9 & 1.3 & 52 \\
\hline$[8]$ & $0.18 \mu \mathrm{m} \mathrm{CMOS}$ & 14 & 10.6 & 47.4 & -11 & -12 & 1.3 & 52 \\
\hline This work & $\mathbf{0 . 1 8} \mu \mathrm{m} \mathrm{CMOS}$ & $\mathbf{3 . 1} \mathbf{1 0 . 6}$ & $\mathbf{1 8}$ & $\mathbf{8 4}$ & $\mathbf{- 1 0}$ & $\mathbf{- 9}$ & $\mathbf{1 . 8}$ & $\mathbf{5 4}$ \\
\hline
\end{tabular}

Table 2 Performances comparison

\section{ACKNOWLEDGEMENT}

The authors would like to acknowledge Chien-Chih Lin, Chia-Hsin Wu, Hua-Chin Lee, Chao-Shiun Wang, and HangChie Tsai for technical discussions. This work was supported in part by Media Tek Inc. HsinChu, Taiwan 300.

\section{REFERENCES}

[1] http://www.ieee802.org/15/pub/TG3a.html

[2] P. J. Sullivan, B. A. Xavier, and W. H. Ku, "An Integrated CMOS Distributed Amplifier Utilizing Packaging Inductance," IEEE Trans. Microwave Theory Tech. , VOL. 45, pp. 1969-1975, Oct. 1997

[3] B. M. Ballweber, R. Gupta and D. J. Allstot, "A Fully Integrated 0.55.5 GHz CMOS Distributed Amplifier," ISSCC, VOL. 35, NO. 2, FEBRUARY 2000

[4] H. T. Ahn and D. J. Allstot, "A $0.5-8.5 \mathrm{GHz}$ Fully Differential CMOS Distributed Amplifier," ISSCC, VOL. 37, NO.8, AUGUST 2002

[5] B. Ballweber, C. H. Diaz, D. Vook, L. Madden, T. H. Lee, and S. S Wong, "Monolithic CMOS Distributed Amplifier and Oscillator," ISSCC Digest of Tech. Papers, 1999, pp. 70-71
[6] A. Worapishet, M. Chongcheawchamnan, and S. Strisathit, "Broadband Amplifier in CMOS Technology using Cascade Singlestage Distributed Amplifier," ELECTRONICS LETTERS, VOL. 38, NO. 14, 4th JULY 2002

[7] R. C. Liu; K. L. Deng; H. Wang, “A 0.6-22-GHz Broadband CMOS Distributed Amplifier" IEEE RFIC Symposium, pp.103 - 106, June 8-10, 2003

[8] R. C. Liu, C. S. Lin, K. L. Deng, and H. Wang, "A 0.5-14-ghz 10.6db CMOS Cascode Distributed Amplifier" Digest of Technical Papers. 2003 Symposium on VLSI Circuits, pp.139 - 141, June 12 14,2003

[9] B. M. Frank, A. P. Freundorfer, Y. M. M. and Antar, "Performance of $1-10-\mathrm{GHz}$ traveling wave amplifiers in $0.18-/ \mathrm{spl} \mathrm{mu} / \mathrm{m}$ CMOS" IEEE Microwave and Wireless Components Letters, VOL. 12, Issue: 9 , pp. $327-329$, Sep 2002

[10] K. Kouznetsov, and A. M. Niknejad, "Design and Analysis of Distributed Amplifier"

[11] Thomas T. Y. Wong, "Fundamentals of Distributed Amplifier"

[12] Welborn, XtremeSpectrum, Inc. Project: IEEE P802.15 Working Group for Wireless Personal Area Networks (WPANs) 